\section{Angiogenesis biomarkers detection on a polymeric 3D printed device}

Gianluca Palmara,1 Alessandro Chiadò, ${ }^{1}$ Annalisa Chiappone,1 Fabrizio Pirri, 1-2 Ignazio Roppolo, ${ }^{1}$ Francesca Frascella ${ }^{1}$ 1Department of Applied Science and Technology, Politecnico di Torino, Turin; 2Sustainable Future Technologies (a)Polito, Istituto Italiano di Tecnologia, Turin, Italy

\section{Abstract}

Polymeric 3D printed chips with intrinsic tuned functionalities were produced and employed for the detection of angiogenesis biomarkers.

\section{Introduction}

The monitoring of tumoral biomarkers involved in pathological angiogenesis is an important task in the establishment of an early diagnostics for patients. Biosensors could represent a promising platform for this purpose. These devices require complex chemical functionalizations to immobilize the biorecognition elements. Here, a novel biosensor consisting in a polymeric 3D-printed chip is presented. The device is printed in a single-step procedure with tuned chemical functionalities, later exploited for the tethering of antibodies. The detection of Vascular Endothelial Growth Factor (VEGF) and Angiopoietin-2 (Ang-2), well-known cancer biomarkers, was performed in order to prove its reliability as a screening tool.

\section{Materials and Methods}

The polymeric chips were realized using a Digital Light Processing 3D printer. ${ }^{1}$ The revealment of the biomarkers of interest and the estimation of their Limit of Detection (LOD) and Limit of Quantification (LOQ) were performed by means of a sandwich ELISA-like immunoassay.

\section{Results}

Acrylic acid was added to three resins, to introduce carboxyl groups in the polymeric matrix, to be exploited as anchor point for the immobilization of any bioreceptor that exposes a primary amine.

The printed polymers were tested for their physicochemical properties and protein grafting capabilities, in order to select the top performing formulation that was afterward used for the production of two Lab-on-Chip devices.

The first chip consisted of a microfluidics responsible to convey samples and buffers to an incubation well, where the specific biomolecular interactions took place. The second chip was an alternative version, with three incubation wells simply accessible through an inlet port (see Figure $1)$.

The LOD evaluated for VEGF and Ang2 were compared with the cut-off values that are reported in the literature. 2,3 VEGF sensitivity was not high enough to reveal abnormal levels of this growth factor. On the other hand, the LOD relative to Ang-2 ( $\left.0.8 \mathrm{ng} \mathrm{mL}^{-1}\right)$ was below the threshold concentration $\left(2.5 \mathrm{ng} \mathrm{mL}^{-1}\right)$, demonstrating a good reliability in a suitable range of values (see Figure 2).
Correspondence: Gianluca Palmara, Department of Applied Science and Technology, Politecnico di Torino, C.so Duca degli Abruzzi 24, 10129Torino, Italy.

Tel.: +39.0110907329

E-mail: gianluca.palmara@polito.it

Key words: Biochemical functionalization; biosensors; 3D-printing; ELISA.

Acknowledgments: This work was performed in the framework and financed by POLITO BIOMed LAB, an interdepartmental laboratory financed by Politecnico di Torino, DEFLeCT project funded by Regione Piemonte and FOOD DRUG FREE project (funded by Regione Piemonte).

Disclosures : There are no conflicts to declare.

Conference presentation: This paper was presented at the Third Centro 3R Annual Meeting - L'era delle 3R: modelli in silico, in vitro e in vivo per promuovere la ricerca traslazionale 30 September - 1 October 2021, Evento online organizzato dal Politecnico di Torino.

Received for publication: 9 July 2021

Accepted for publication: 7 September 2021.

This work is licensed under a Creative Commons Attribution NonCommercial 4.0 License (CC BY-NC 4.0).

CCopyright: the Author(s), 2021

Licensee PAGEPress, Italy

Biomedical Science and Engineering 2021; 4(s1):186 doi:10.4081/bse.2021.186

\section{Discussion and Conclusions}

The combination of the 3D-printing technology with an improvement of photocurable formulations allowed to design a polymeric device having intrinsic tuned

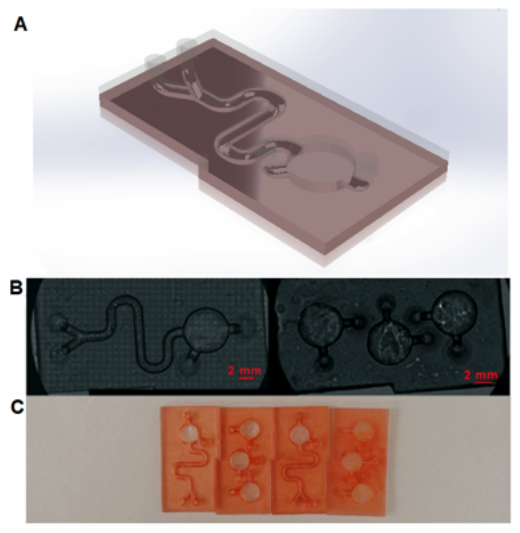

Figure 1. A) CAD model of the LOC; B) microscope images of the $3 \mathrm{D}$ printed microfluidic device; $\mathrm{C}$ ) images of the printed modular chip.

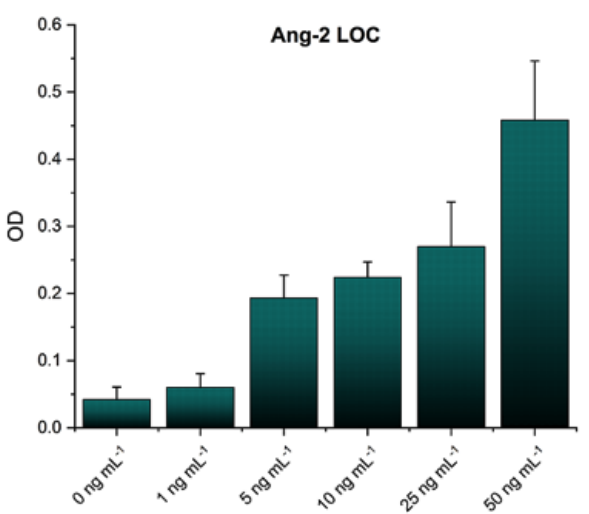

Figure 2. Detection of Ang-2 through sandwich ELISA-like protocol in the modular LOC: the graph shows the OD signals obtained for different concentrations of Ang-2. 


\section{Article}

functionalities with no need for further chemical derivatization. This system was able to specifically recognize Ang-2 with a LOD compatible with the range of concentrations that are required for the identification of a malignant scenario.

\section{References}

1. Chiadò A, Palmara G, Chiappone A, et al. A modular 3D printed lab-on-a-chip for early cancer detection. Lab Chip 2020;20:665-74.

2. Monastero RN, Pentyala S. Cytokines as biomarkers and their respective clinical cutoff levels. Int J Inflam 2017;2017:4309485.

3. Li P, He Q, Luo C, Qian L. Diagnostic and prognostic potential of serum angiopoietin-2 expression in human breast cancer. Int $\mathrm{J}$ Clin Exp Pathol 2015;8:660-4 